\title{
Lack of PAH emission toward low-mass embedded young stellar objects ${ }^{\star}$
}

\author{
V. C. Geers ${ }^{1,2}$, E. F. van Dishoeck ${ }^{1}$, K. M. Pontoppidan ${ }^{3}$, F. Lahuis ${ }^{4}$, A. Crapsi ${ }^{1,5}$, C. P. Dullemond ${ }^{6}$, and G. A. Blake ${ }^{3}$
}

1 Leiden Observatory, Leiden University, PO Box 9513, 2300 RA Leiden, The Netherlands

2 University of Toronto, 50 St. George St., Toronto, ON M5R 2W9, Canada e-mail: vcgeers@astro.utoronto.ca

3 Division of Geological and Planetary Sciences, Mail Code 150-21, California Institute of Technology, Pasadena, CA 91125, USA

4 SRON Netherlands Institute for Space Research, PO Box 800, 9700 AV Groningen, The Netherlands

5 Observatorio Astronómico Nacional (IGN), Alfonso XII, 3, 28014 Madrid, Spain

6 Max-Planck-Institut für Astronomie, Koenigstuhl 17, 69117 Heidelberg, Germany

Received 19 September 2008 / Accepted 2 December 2008

\section{ABSTRACT}

\begin{abstract}
Aims. Polycyclic aromatic hydrocarbons (PAHs) have been detected toward molecular clouds and some young stars with disks, but have not yet been associated with embedded young stars. We present a sensitive mid-infrared spectroscopic survey of PAH features toward a sample of low-mass embedded young stellar objects (YSOs). The aim is to put constraints on the PAH abundance in the embedded phase of star formation using radiative transfer modeling.

Methods. VLT-ISAAC $L$-band spectra for 39 sources and Spitzer IRS spectra for 53 sources are presented. Line intensities are compared to recent surveys of Herbig Ae/Be and T Tauri stars. The radiative transfer codes RADMC and RADICAL are used to model the PAH emission from embedded YSOs consisting of a pre-main-sequence star with a circumstellar disk embedded in an envelope. The dependence of the PAH feature on PAH abundance, stellar radiation field, inclination and the extinction by the surrounding envelope is studied.

Results. The $3.3 \mu \mathrm{m}$ PAH feature is undetected for the majority of the sample (97\%), with typical upper limits of $5 \times 10^{-16} \mathrm{~W} \mathrm{~m}^{-2}$. One source originally classified as class I, IRS 48, shows a strong $3.3 \mu \mathrm{m}$ feature from a disk. Compact $11.2 \mu \mathrm{m}$ PAH emission is seen directly towards 1 out of the 53 Spitzer Short-High spectra, for a source that is borderline embedded. For all 12 sources with both VLT and Spitzer spectra, no PAH features are detected in either. In total, PAH features are detected toward at most 1 out of 63 (candidate) embedded protostars $(\$ 2 \%)$, even lower than observed for class II T Tauri stars with disks (11-14\%). Models predict the $7.7 \mu \mathrm{m}$ feature as the best tracer of PAH emission, while the $3.3 \mu \mathrm{m}$ feature is relatively weak. Assuming typical class I stellar and envelope parameters, the absence of PAH emission is most likely explained by the absence of emitting carriers through a PAH abundance at least an order of magnitude lower than in molecular clouds but similar to that found in disks. Thus, most PAHs likely enter the protoplanetary disks frozen out in icy layers on dust grains and/or in coagulated form.
\end{abstract}

Key words. stars: pre-main sequence - stars: planetary systems: protoplanetary disks - stars: circumstellar matter - astrochemistry

\section{Introduction}

Polycyclic aromatic hydrocarbons (PAHs) have been observed toward a wide range of astrophysical environments (Allamandola et al. 1989; Peeters et al. 2002), including the interstellar medium (ISM) and star-forming regions, first hinted at by the discovery of widespread broad emission features in the mid-infrared. Since PAHs are a good tracer of UV radiation, they are an indirect probe of star formation in the high opacity environments of molecular clouds as well as circumstellar disks. Recent spectroscopic studies have detected PAHs toward a significant fraction, $11-14 \%$ and $54 \%$ of low-mass and intermediate mass pre-main-sequence (PMS) stars respectively (Acke \& van den Ancker 2004; Geers 2007, Chap. 2), and recent ground-based high spatial resolution observations show evidence that the PAH emission originates from the circumstellar disks (Ressler \& Barsony 2003; Habart et al. 2006; Geers et al. 2007a,b). PAHs are also prominently seen toward photon dominated regions (PDRs) in dense clouds exposed to massive young

* Based on observations obtained at the European Southern Observatory, Paranal, Chile, within the observing program 164.I-0605 (VLT-ISAAC). stars (e.g., Verstraete et al. 1996). However, to this date, no significant PAH emission has been reported to be directly associated with the earlier class $0-$ I phase, when a (proto)star with a circumstellar disk is still embedded in an envelope of gas and dust. Deeply embedded high-mass young stellar objects (YSOs) also lack prominent PAH emission (van Dishoeck \& van der Tak 2000).

PAHs are believed to form in the outflows of carbon-rich asymptotic giant branch (AGB) stars, which deposit them in the interstellar medium (e.g., Helling et al. 1996). Their ubiquitous presence in the ISM shows that at least larger PAH molecules (of 50-100 carbon atoms or more) will survive this phase, until being incorporated in molecular clouds. The amount of carbon locked up in PAHs is $[\mathrm{C} / \mathrm{H}]_{\mathrm{PAH}} \simeq 5 \times 10^{-5}$ (Habart et al. 2004), corresponding to $\mathrm{PAH}$ abundances of $\sim 5 \times 10^{-7}$ relative to $\mathrm{H}$, for PAH molecules of 100 carbon atoms, making them among the most abundant molecules after $\mathrm{H}_{2}$ and $\mathrm{CO}$.

PAHs can play an important role in the star-forming environments. As large molecules, they provide efficient heating of the gas in both the ISM and circumstellar disks. Small dust particles like PAHs have also been suggested as the main formation site of molecules such as $\mathrm{H}_{2}$ and water in the later evolutionary phases 
of circumstellar disks when classical grains have grown to large sizes (Jonkheid et al. 2006).

The shape and relative strength of the PAH features in the 6-9 $\mu \mathrm{m}$ region has been shown to vary between various astrophysical environments from AGBs and the ISM to the class II disk sources. This has provided evidence that the emission characteristics of PAHs are sensitive to local physical conditions and that interstellar PAHs undergo processing in space environments (Peeters et al. 2002). Further support for this comes from recent values for the PAH abundance in disk surface layers, which is a factor of 10-100 lower than in the ISM for sources with sufficient UV radiation to excite them (Geers et al. 2006). PAHs frozen out on icy grains can chemically react with other molecules to form a large variety of complex species, including pre-biotic molecules (Bernstein et al. 1999; Ehrenfreund et al. 2006). A study of PAHs toward embedded low-mass young stars is important to find out what happens to PAHs and how large a role they may play in these embedded objects.

In this paper we present the first mid-infrared spectroscopic survey for PAH emission from embedded low-mass young stellar objects using the Infrared Spectrometer And Array Camera (ISAAC) mounted on the ESO Very Large Telescope (VLT) and the Infrared Spectrograph (IRS) on board the NASA Spitzer Telescope, in a wide range of nearby star-forming regions. We subsequently use a radiative transfer code to model PAH emission from young stars with disks embedded in an envelope, and discuss the observed detection rate in the context of PAH abundance, the strength of UV emission from the protostar and the extinction from the envelope.

\section{Observations and data reduction}

A large sample of low-mass embedded objects was selected from two previous mid-infrared studies. A set of 39 sources was selected from a VLT-ISAAC 3-5 $\mu \mathrm{m}$ band survey (van Dishoeck et al. 2003), of which 32 were previously presented in Pontoppidan et al. (2003) and three additional sources in Thi et al. (2006). For this survey $L$-band spectroscopy was obtained with ISAAC, the Infrared Spectrometer And Array Camera, installed at the VLT Antu (UT1) at ESO's Paranal Observatory in Chile, in the low resolution $(R=\lambda / \Delta \lambda=600$ 1200) spectroscopic mode in the spectral domain $2.8-4.2 \mu \mathrm{m}$ using a $0.6^{\prime \prime} \times 120^{\prime \prime}$ slit. The telescope was operated using a standard chop-throw scheme with typical chop-throws of 10 $20^{\prime \prime}$. A full description of observation and reduction techniques is given in Pontoppidan et al. (2003). These sources were required to have a rising spectral energy distribution (SED) in the mid-infrared as well as the presence of an $\mathrm{H}_{2} \mathrm{O}$ ice feature at $3 \mu \mathrm{m}$. The selected sources are listed in Table A.1.

In addition, a sample of 53 Spitzer Space Telescope Infrared Spectrograph (Spitzer IRS) Short-High (10-20 $\mu \mathrm{m}, R \sim 600)$ spectra and 33 Short-Low $(5-14.5 \mu \mathrm{m}, R \sim 100)$ of class I sources were obtained in the context of the "Cores to Disks" (c2d) Legacy program (Evans et al. 2003). These sources were selected as showing the silicate $10 \mu \mathrm{m}$ feature in absorption, and the list includes a small number of known or candidate edgeon disks, which are labelled in Table A.1. Most of them have spectral slopes $\alpha$ between 2 and $24 \mu \mathrm{m}>0$. The vast majority of class 0 and I sources in the c2d sample were found to have luminosities of $L_{\mathrm{bol}}<3 L_{\odot}$ (Evans et al. 2008) indicative of low mass young stars and consistent with the observational fact that the majority of the young stars in the regions studied by c2d are K- and M-type stars (Luhman 2007; Oliveira et al. 2008). The reduction of the Spitzer spectroscopy was performed using the optimal PSF extraction technique developed within the c2d program, to allow separation of the compact source and extended emission components (Lahuis et al. 2007). The source size is determined from the width of the PSF function fitted to the source, compared to the width of the PSF function fit for standard calibrator stars. Comparison of the optimal PSF extraction and the full aperture extraction provides a direct estimate of any potential extended emission. An application of the same technique to remove background PAH features has been given for the source VSSG 1 in Geers et al. (2006, see their Fig. 4). The details of the observations and reduction procedures are described in Lahuis et al. (2006) and Lahuis (2007, thesis Chap. 3).

In total, the sample contains 80 sources, for which 12 sources were observed with both ISAAC and Spitzer. Based on new 2MASS-Spitzer-MIPS 2-24 $\mu \mathrm{m}$ spectral slopes and/or sub-mm line and continuum data, as well as $\mathrm{CO} 4.7 \mu \mathrm{m}$ spectroscopy, the classification has been refined (van Kempen et al. 2008; Enoch et al. 2008; van Kempen 2008; Jørgensen et al. 2008, Pontoppidan priv. comm.). These updates have confirmed 53 embedded sources and 17 sources to be disk sources, while for 10 sources the classification remains uncertain (Table A.1).

\section{Results and discussion}

\subsection{VLT-ISAAC spectra}

The VLT-ISAAC $L$-band spectra are presented in Fig. 1. Only 1 source in this sample shows a clear $3.3 \mu \mathrm{m}$ PAH emission feature, IRS 48. This source is known to have a spatially resolved disk surrounded by a very tenuous envelope at most (Geers et al. 2007a). No clear PAH feature is seen toward any of the other sources. To derive upper limits, a Gaussian with a peak flux equal to the $3 \sigma$ noise and $F W H M=0.07 \mu \mathrm{m}$ is generated and integrated to retrieve the line flux of the largest PAH $3.3 \mu \mathrm{m}$ feature that would still fall within the noise. The results are listed in Table A.1.

The hydrogen Pf $\delta(3.296 \mu \mathrm{m})$, Pf $\gamma(3.739 \mu \mathrm{m})$ and $\operatorname{Br} \alpha$ $(4.051 \mu \mathrm{m})$ are detected toward most sources, with the exception of IRAS 11068-7717 and Cha IRN. These lines are believed to be produced in the accretion column onto the central star (Calvet \& Gullbring 1998), and/or in the wind launched close to the star (Kraus et al. 2008). The broad absorption features by water ice $(2.8 \mu \mathrm{m})$ and the $3.47 \mu \mathrm{m}$ ice feature can be clearly seen toward the majority of the sources.

\subsection{Spitzer spectra}

Out of the sample of 53 sources, 50 do not show any PAH features. A selection of Spitzer spectra with PAH non-detections is presented in Fig. 2. Silicate, water and $\mathrm{CO}_{2}$ absorption are the predominant features in these spectra. These have been analyzed by Boogert et al. (2008) and Pontoppidan et al. (2008).

For one Spitzer source, Oph GY 23, the optimal extraction method produces an SH spectrum with the observed $11.2 \mu \mathrm{m}$ PAH features, shown in Fig. 3. A small size is derived for this source, suggesting that the PAH emission is directly associated with the star. The classification of this source remains uncertain. Based on Spitzer IRS, IRAC and MIPS fluxes, Oph GY 23, previously classified as class I, shows a declining SED suggestive of a class II source. However, Oph GY 23 is surrounded by reflection nebulosity and deep hot $\mathrm{CO}$ gas absorption features have been observed towards this source (Pontoppidan, priv. comm.), hinting at the presence of remnant envelope material. 


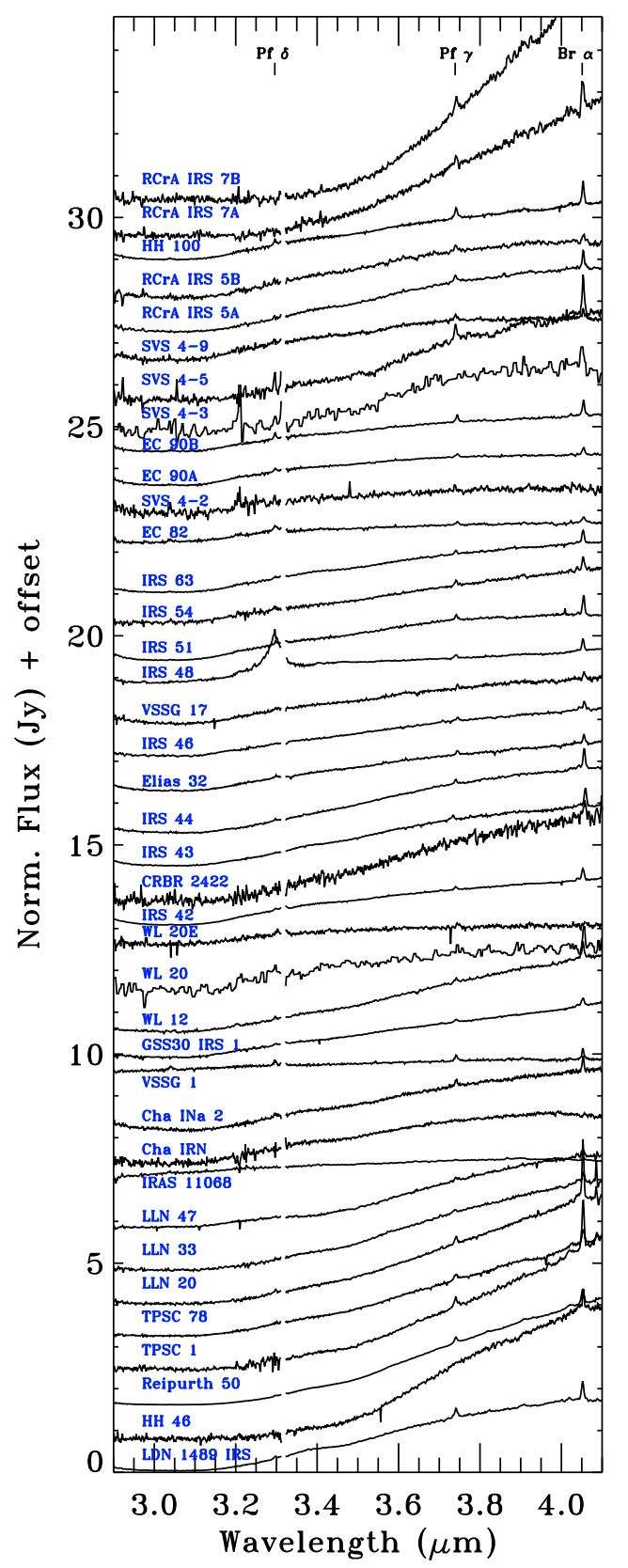

Fig. 1. ISAAC $L$-band spectra of low-mass embedded sources. The narrow $\mathrm{HI}$ lines at 3.3, 3.74 and $4.05 \mu \mathrm{m}$ are seen in most spectra.

For two sources, VSSG 1 and IRS 14 in Ophiuchus, PAH features are detected from the extended background emission, which is assumed not to be directly associated with a central object.

Overall, no PAHs are detected toward any of the 53 confirmed embedded sources. PAHs are detected towards one of the 17 confirmed disk sources, IRS 48, and one out of the 10 sources with an uncertain classification, Oph GY 23.

\section{Radiative transfer model}

Here we address the question of where the PAH emission can arise in a disk + envelope system through radiative transfer modeling and what the non-detections imply quantitatively about the PAH abundance.

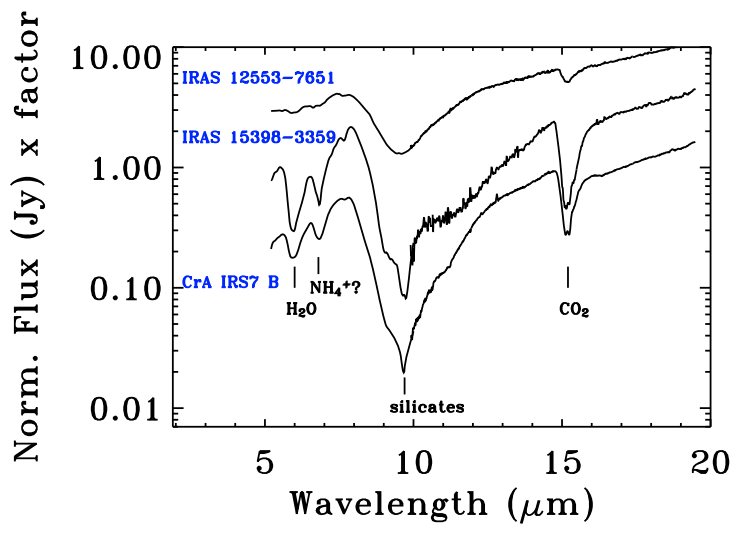

Fig. 2. Spitzer IRS spectra of a sample of low-mass embedded sources without PAH detections. For clarity, the spectra are normalized to their flux at $17 \mu \mathrm{m}$ and scaled by factors of 1,3 and 8 for CrA IRS7 B, IRAS 15398-3359 and IRAS 12553-7651 respectively.

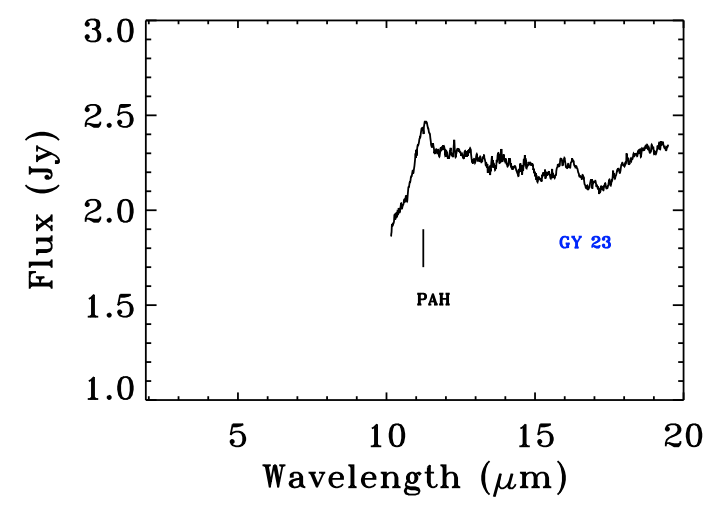

Fig. 3. Spitzer IRS spectra of Oph GY 23, a low-mass borderline embedded source with a PAH detection.

\subsection{Physical structure}

We use the two-dimensional axisymmetric radiative transfer code RADMC (Dullemond \& Dominik 2004) to calculate the temperature structure and scattering source function for an embedded YSO using a Monte Carlo technique. This code requires stellar parameters, a stellar radiation field, a density structure and a set of dust opacities. Scattering is assumed to be isotropic. A module to treat the emission from quantum-heated PAH molecules and Very Small Grains, previously described in Geers et al. (2006), has been included. To generate images and spectra, ray-tracing is performed using RADICAL (Dullemond $\&$ Turolla 2000). All spectra and SEDs presented here are scaled to a distance to the observer of $150 \mathrm{pc}$.

The density structure adopted here follows Crapsi et al. (2008) and is comprised of three components: the disk, the envelope and the outflow cone. The adopted density structure for the disk has a power-law dependence along the radial coordinate and a Gaussian dependence in height, and can be expressed as

$\rho_{\text {disk }}(r, \theta)=\frac{\Sigma_{0}\left(r / R_{0}\right)^{-1}}{\sqrt{2 \pi} H(r)} \exp \left\{-\frac{1}{2}\left[\frac{r \cos \theta}{H(r)}\right]^{2}\right\}$,

where $\theta$ is the angle from the axis of symmetry. The variation of scale-height, i.e., the flaring of the disk, is described in the 
Table 1. Parameters of the template model.

\begin{tabular}{ll}
\hline \hline Parameter & Value \\
\hline Radiation field & $4000 \mathrm{~K}$ blackbody \\
& + excess UV field \\
$L_{*}$ & $1 L_{\odot}$ \\
$M_{*}$ & $1.0 M_{\odot}$ \\
$M_{\text {disk }}$ & $5 \times 10^{-2} M_{\odot}$ \\
$R_{\text {disk,in }}$ & $0.1 \mathrm{AU}$ \\
$R_{\text {disk,out }}$ & $300 \mathrm{AU}$ \\
$M_{\text {env }}$ & $1.0 M_{\odot}$ \\
$R_{\text {env,out }}$ & $10000 \mathrm{AU}$ \\
$R_{\text {rot }}$ & $300 \mathrm{AU}$ \\
$\theta_{0}$ & $15^{\circ}$ \\
PAH abun. & $5 \times 10^{-7}$ w.r.t. H \\
\hline
\end{tabular}

function $H(r)=r \cdot H_{0} / R_{0} \cdot\left(r / R_{0}\right)^{2 / 7}$, corresponding to the selfirradiated passive disk of Chiang \& Goldreich (1997). For the disk in our template model, we fix the outer radius $R_{0}=300 \mathrm{AU}$, inner radius $R_{\text {in }}=0.1 \mathrm{AU}, H_{0}=90 \mathrm{AU}$, and the disk mass to $M_{\text {disk }}=5 \times 10^{-3} M_{\odot}$, see also Table 1 .

The envelope density follows the theoretical structure for a rotating and collapsing spheroid as derived by Ulrich (1976), defined by

$$
\begin{aligned}
\rho_{\mathrm{env}}(r, \theta)= & \rho_{0}\left(\frac{R_{\mathrm{rot}}}{r}\right)^{1.5}\left(1+\frac{\cos \theta}{\cos \theta_{0}}\right)^{-0.5} \\
& \times\left(\frac{\cos \theta}{2 \cos \theta_{0}}+\frac{R_{\mathrm{rot}}}{r} \cos ^{2} \theta_{0}\right)^{-1},
\end{aligned}
$$

where $\theta_{0}$ is the solution of the parabolic motion of an infalling particle which is given by $r / R_{\text {rot }} \cdot\left(\cos \theta_{0}-\cos \theta\right) /\left(\cos \theta_{0} \sin ^{2} \theta_{0}\right)=$ $1, R_{\text {rot }}$ is the centrifugal radius of the envelope, and $\rho_{0}$ is the density of the equatorial plane at the centrifugal radius. The outer radius of the envelope is fixed to $10000 \mathrm{AU}$ and the centrifugal radius is set to $300 \mathrm{AU}$. The value of $\rho_{0}$ is varied to cover a range of envelope mass $M_{\text {env }}$ between 0.1 and $1.5 M_{\odot}$, the template model has $M_{\text {env }}=1.0 M_{\odot}$. This is somewhat more massive than the average Class I source, which typically has $M_{\text {env }}<0.5 M_{\odot}$. The inner radius of the envelope is determined by the outflow cavity, which crosses the equatorial plane at $\sim 20 \mathrm{AU}$. The streamline outflow is included by setting the density of the regions where $\cos \theta_{0}$ is larger than $\cos 15^{\circ}$ to the same value of the density of the envelope at the outer radius. This results in a funnel-shaped cavity which is conical only at large scales, where it presents a semi-aperture of $15^{\circ}$. The envelope introduces an optical extinction of $A_{\mathrm{V}}=33$ at $i=45^{\circ}$, for the template model with $M_{\text {env }}=1.0 M_{\odot}$.

For the radiation field, a blackbody spectrum is taken with $T_{\text {eff }}=4000 \mathrm{~K}$ and a luminosity of $1 L_{\odot}$ for the template model. In addition, given the strong dependence of PAHs on UV excitation and observations indicating the presence of UV excess around actively accreting young low-mass PMS stars (Basri \& Bertout 1989; Hartmann \& Kenyon 1990), the radiation field for $\lambda<0.8 \mu \mathrm{m}$ is substituted by a modified Draine field (van Dishoeck \& Black 1982), scaled up to ensure continuous overlap at $\lambda>0.8 \mu \mathrm{m}$. The resulting spectrum is normalized to a luminosity of $1 L_{\odot}$. For comparison, a separate model is run without the modified radiation field. Any radiation field produced by a higher mass (A-type) star would be in between these two extremes. The excess Draine UV field corresponds to an intensity relative to the mean interstellar radiation field, $G_{0}$, of $8 \times 10^{4}$ at a radius of $100 \mathrm{AU}$ at the surface of the disk.

\subsection{Treatment of dust and PAHs}

For the optical properties of the dust grain population, we adopt the set of dust mixtures and opacities used in Crapsi et al. (2008): a mixture of $71 \%$ silicates with a size distribution similar to that of Weingartner \& Draine (2001) with the $\alpha_{\mathrm{WD}}$-parameter equal to -2 and turnover point for radii larger than $0.3 \mu \mathrm{m}$ covered by a mantle of ices with a water ice abundance of $3.0 \times 10^{-4}$ relative to $\mathrm{H}_{2}$, together with $29 \%$ carbonaceous grains with a size distribution similar to that of Weingartner \& Draine (2001), characterized by a turnover point at radius of $4.5 \mu \mathrm{m}$, an $\alpha_{\mathrm{WD}}$-parameter of -2 and a water ice abundance of $1.5 \times 10^{-5}$ relative to $\mathrm{H}_{2}$. A second dust population is added without the ice layer, for regions where the temperature is higher than $90 \mathrm{~K}$, when ices will have evaporated. These are included by running the Monte Carlo code once with ices in the whole grid to calculate the temperature structure, and then using this information to replace the dust opacities where the temperature is higher than $90 \mathrm{~K}$. The ice features include $\mathrm{H}_{2} \mathrm{O}, \mathrm{CO}_{2}$ and $\mathrm{CO}$, but not more complex species which could be responsible for (part of) the observed 6.0 and $6.8 \mu \mathrm{m}$ ice features (Boogert et al. 2008).

PAHs are added as a dust species to the disk and the envelope. The PAH emission is calculated for an equal mix of neutral and singly ionized $\mathrm{C}_{100} \mathrm{H}_{24}$ molecules, adopting the Draine \& $\mathrm{Li}$ (2001) PAH emission model, using the "thermal continuous" approximation. Multi-photon events are included for the PAH excitation, following the method outlined by Siebenmorgen et al. (1992). We include the opacities from Mattioda et al. (2005) for near-infrared wavelengths. Model calculations by Visser et al. (2007) show that PAHs with $N_{\mathrm{c}}=100$ have longer lifetimes than the lifetime of the disk, and we can therefore safely discard the possibility of PAH destruction and keep the PAHs at constant abundance throughout the entire disk.

In our template model, the total $\mathrm{PAH}$ abundance is the same as used in Geers et al. (2006), as a mass fraction of the dust of 0.061 , which corresponds to a carbon abundance of $5 \times 10^{-5}$ with respect to hydrogen and a $N_{\mathrm{c}}=100 \mathrm{PAH}$ abundance of $5 \times 10^{-7}$ with respect to hydrogen. This PAH abundance is divided into 50\% ionized and 50\% neutral PAHs, the same as in our previous disk-only models (Geers et al. 2006, 2007b).

A spectrum of the template model, with and without PAHs, is shown in Fig. 4. The main features in the spectrum are the PAH features at 3.3, 6.2, 7.7, 8.6, 11.2 and $12.7 \mu \mathrm{m}$, as well as water, $\mathrm{CO}$, silicate and $\mathrm{CO}_{2}$ ice absorption features at 3.08 , 4.67, 9.7 and $15.2 \mu \mathrm{m}$ respectively. The wavelength coverage of the ISAAC L-band and Spitzer IRS spectroscopy is indicated.

\subsection{Modeling results}

The apparent absence of PAH features toward the majority of low-mass embedded class I sources could have a number of explanations.

First, PAH molecules are primarily excited by UV photons. The precise shape and strength of the radiation field inside an embedded object is not well-known but in the class I sources the central source has already formed and is the main energy source inside the envelope. The presence or absence of excess UV will influence the PAH emission features. In particular, the high opacity of the envelope at UV and optical wavelengths, which 


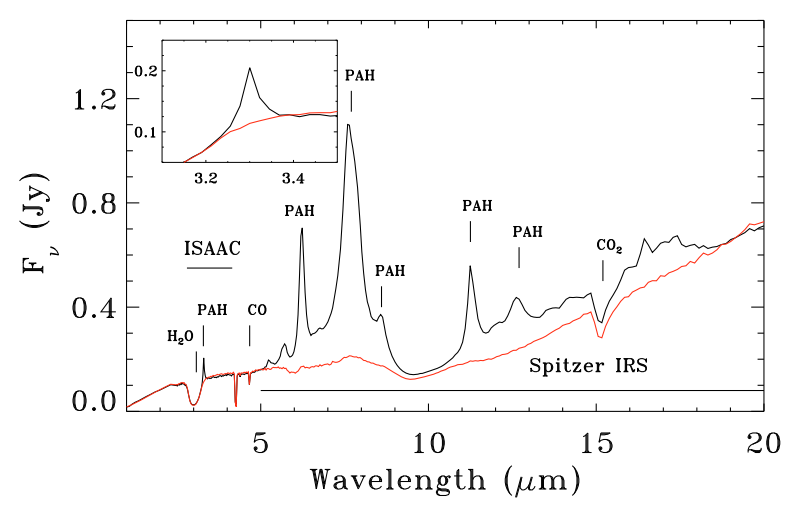

Fig. 4. Model spectra at $i=45^{\circ}$ for template parameters listed in Table 1, with PAHs (black) and without PAHs (red). Major PAH and absorption features are indicated, as well as the wavelength coverage of the presented ISAAC and Spitzer IRS observations. The detail shows a blow-up of the $3.3 \mu \mathrm{m}$ feature on the red wing of the water absorption band.

provide the main excitation of PAHs, will constrain the region where PAHs can be excited to a small radius.

Second, even if the infrared PAH features are produced in the disk or inner envelope close to the star, the dust in the surrounding envelope may provide too high extinction in the optical and mid-infrared for the (PAH) emission to escape, especially if the envelope mass is (still) relatively high compared to the disk. If an outflow cavity is present, the inclination at which the object is observed will affect the extinction by the envelope.

Third, the abundance of small PAH molecules in the gas phase may be significantly lower due to freeze-out due to the low temperatures and high densities in the interior of the molecular core.

In practice, a combination of the above three causes will apply simultaneously. To estimate their effects, models are run, varying several parameters including radiation field, PAH abundance and the mass of the envelope.

\subsubsection{Luminosity of the central source and presence of UV excess}

Models with total stellar luminosity $L_{*}$ varying between 1,3 and $6 L_{\odot}$ are shown in Fig. 5. In addition, a model without the Draine field for UV excess is shown for $L_{*}=6 L_{\odot}$. Increasing the luminosity by a factor of 6 increases the line flux of the PAH features by the same amount while the PAH feature / continuum ratio is reduced by at most $20 \%$. Excluding the excess UV field, while preserving the total stellar luminosity, decreases the PAH feature / continuum ratios by $\sim 3$. Interestingly, the $3.3 \mu \mathrm{m}$ feature is affected most because it requires higher energies to be excited than the other features. For typical luminosities observed toward lowmass embedded protostars, PAH features should be detectable if they have ISM abundances, even if no UV excess is present.

\subsubsection{Mass of the envelope}

Models with the mass of the envelope $M_{\text {env }}$ varying from 0.1 , $0.5,1.0$ to $1.5 M_{\odot}$ are shown in Fig. 6 , at $i=45^{\circ}$. In all models the PAH features are clearly present. Increasing the envelope mass by a factor of 15 results in a decrease of the emission of the central source while the sub-mm emission and the strength of the absorption features increase. The PAH features decrease
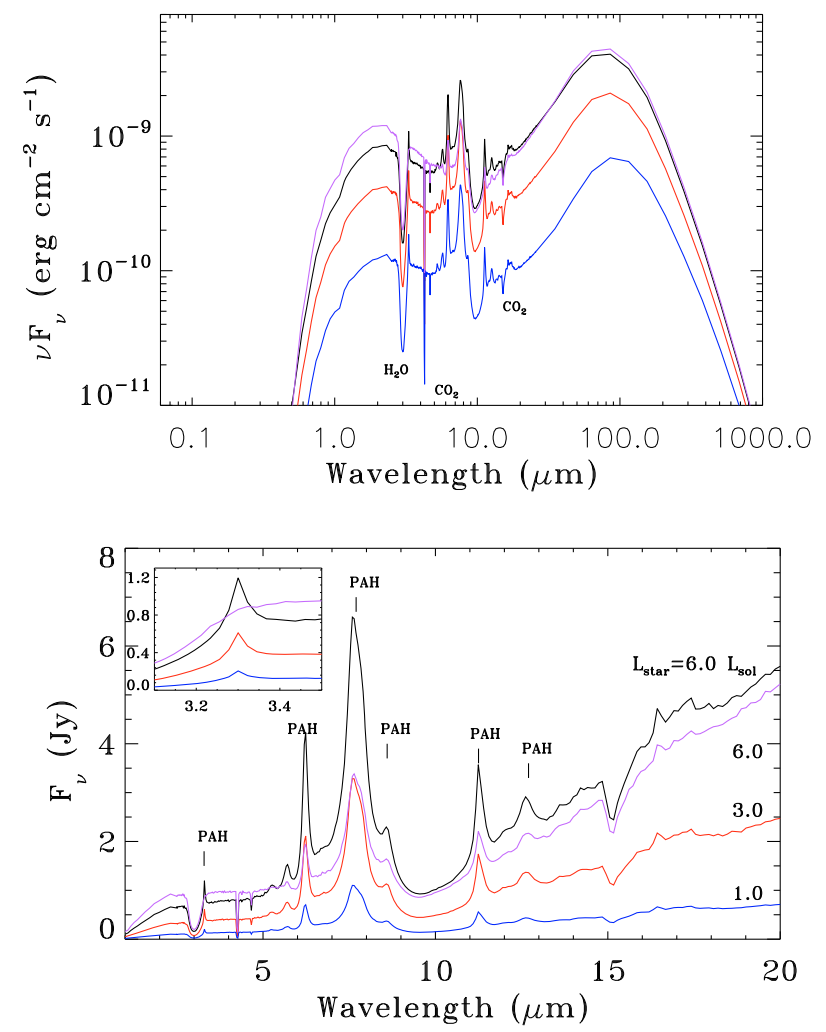

Fig. 5. Model SEDs (top) and blow-up of spectra (bottom) at $i=45^{\circ}$ for template model parameters and $L_{*}$ varying between 6, 3, and $1 L_{\odot}$ (black, red, blue respectively), all including UV excess. A model with $L_{*}=6 L_{\odot}$ without UV excess is shown in purple. Major PAH and absorption features are indicated. The detail shows a blow-up of the $3.3 \mu \mathrm{m}$ feature on the red wing of the water absorption band.

in peak flux by a factor of $3-4$, but are in no case extinguished by the continuous extinction or silicate absorption features. This is consistent with the conclusions of Manske \& Henning (1999) for higher mass YSOs.

In the template models, PAHs are located in both the envelope and the disk. To test the influence of the envelope further, model setups including PAHs only in the disk or only in the envelope were performed. A comparison is shown in Fig. 7, for $M_{\text {env }}=1.0$ and $0.1 M_{\odot}$. Including PAHs only in the disk results in a decrease of peak flux of the PAH features by a factor $\sim 2$. In the SED, the absence of PAHs in the envelope leads to less absorption of UV emission, which appears stronger. The mass of the envelope has no significant effect on the strength of the PAH features, although the underlying continuum changes. Including PAHs only in the envelope also results in a typical decrease of the PAH feature peak flux, by about a factor of 1.5. Even if the PAHs are located only in the envelope, PAHs should be detectable if they are present at ISM abundance.

\subsubsection{Outflow cavity and inclination}

Embedded class I objects are known to have outflows (e.g., Hogerheijde et al. 1998; Arce \& Sargent 2006), thus an outflow cavity is included in our model envelope. Because of the presence of the outflow cavity, the inclination at which the object is observed is important, because at near-pole-on orientation one directly observes the central source and the disk. At larger inclinations, the envelope will be obscuring the disk. 

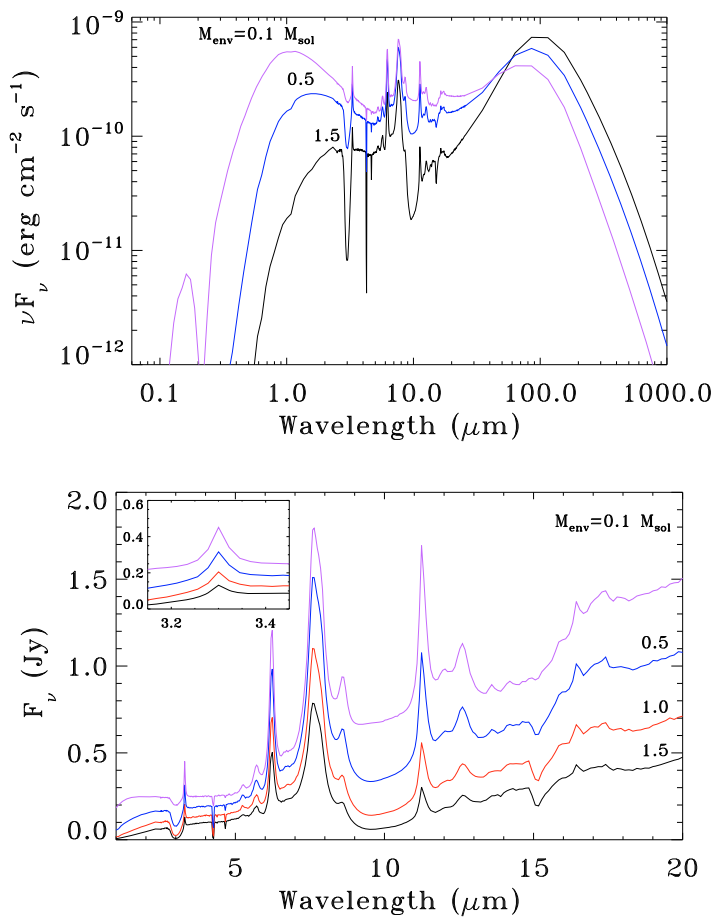

Fig. 6. Model SEDs (top) and blow-up of spectra (bottom) with $M_{\mathrm{env}}=$ 1.5 (black), 1.0 (red), 0.5 (blue) and 0.1 (purple) $M_{\odot}$ at $i=45^{\circ}$. The curve for $1.0 M_{\odot}$ was omitted from the SED plot for clarity. The detail shows a blow-up of the $3.3 \mu \mathrm{m}$ PAH feature.
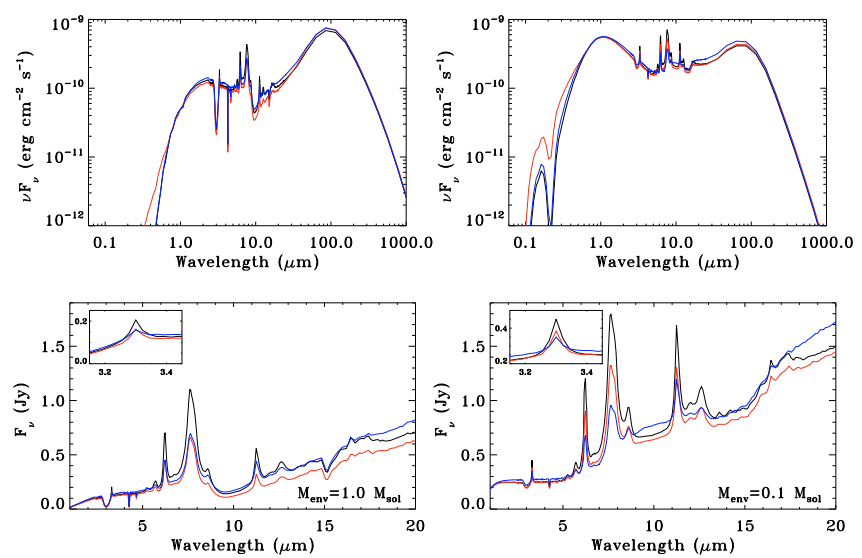

Fig. 7. Model SEDs (top) and blow-up of spectra (bottom) with PAHs in both envelope and disk (black), only in the disk (red) and only in the envelope (blue), at $i=45^{\circ}$, for $M_{\mathrm{env}}=1.0$ (left) and $0.1 M_{\odot}(r i g h t)$. The detail shows a blow-up of the $3.3 \mu \mathrm{m}$ PAH feature.

A template model with the template PAH abundance and an envelope mass of $1.0 M_{\odot}$ seen at varying inclination angles between 5 and $85^{\circ}$ is shown in Fig. 8. At an inclination of $i=5^{\circ}$ the stellar radiation field (blackbody + scaled Draine field) is directly visible and no absorption features are present. Between inclinations of $5^{\circ}$ and $25^{\circ}$, i.e., down the cavity and through the envelope, the appearance of the SED and the PAH features changes rapidly (e.g., Whitney et al. 2003; Crapsi et al. 2008). The emission of the central star becomes obscured and the strength of the PAH features decreases by about a factor of 2 . At increasing inclination the PAH features become weaker, but still dominate the spectrum. Ice absorption features can be seen at 3, 4.2, 6 and $15 \mu \mathrm{m}$. At $85^{\circ}$, the disk is observed almost edge-on and the PAH
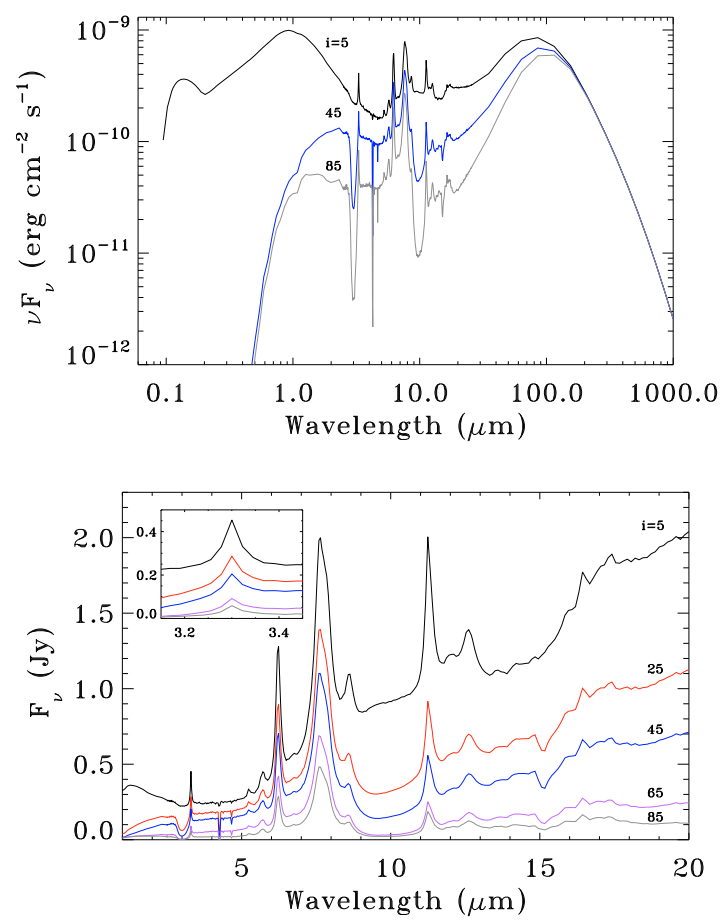

Fig. 8. Model SEDs (top) and blow-up of spectra (bottom) for $i=5,25$, 45,65 and $85^{\circ}$ (black, red, blue, purple, gray, respectively). Curves for $i=25$ and $65^{\circ}$ were omitted from the SED plot for clarity. The detail shows a blow-up of the $3.3 \mu \mathrm{m}$ PAH feature.

emitting regions are largely masked by the disk itself. The remaining features in the spectrum extracted for an infinitely large aperture are due to scattered emission, originating from higher up in the disk atmosphere. Observed PAH feature strengths will depend on the pointing and orientation of the limited aperture slit on the embedded source.

\subsection{4. $\mathrm{PAH}$ abundance}

Models with the total PAH abundance (both ionized and neutral species) varying between the template abundance, and factors of 10, 20 and 100 lower, are shown in Fig. 9. The envelope mass in these models is $1.0 M_{\odot}$. Reducing the PAH abundance by a factor of 10 has the straightforward result of reducing the PAH peak flux by a factor of 3 . The $3.3 \mu \mathrm{m}$ feature is notably weak in all models, and already at a factor of 10 lower abundance is no longer detectable. Decreasing the PAH abundance also leads to an increase in the UV emission and continuum emission at farIR wavelengths. The strongest feature, $7.7 \mu \mathrm{m}$, is visible down to an abundance of $1 \times 10^{-8}$ relative to $\mathrm{H}$, i.e., a value $50 \mathrm{x}$ lower than the ISM.

\subsection{Summary and caveats}

We find in all models that the $7.7 \mu \mathrm{m}$ feature is noticeably the strongest PAH feature, with the highest feature to continuum ratio. This is in sharp contrast with observational and modeling results for non-embedded class II stars with disks, where the silicate emission feature largely obscures the contribution of the 7.7 and $8.6 \mu \mathrm{m}$ features. There is no readily visible $7.7 \mu \mathrm{m} \mathrm{PAH}$ feature in our observations. Increasing the envelope mass and decreasing PAH abundance, luminosity or UV excess all lead to weaker PAH features. The inclination has the largest influence 

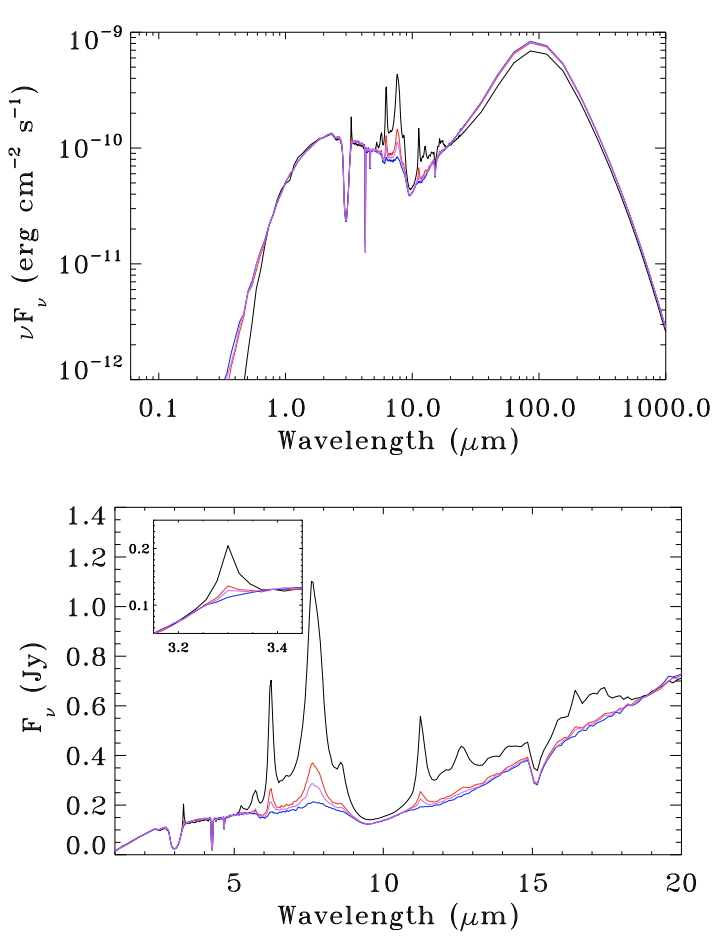

Fig. 9. Model SEDs (top) and blow-up of spectra (bottom) with template PAH abundance (black), and factors of 10 (red), 20 (purple) and 100 lower (blue) at $i=45^{\circ}$. The detail shows a blow-up of the $3.3 \mu \mathrm{m} \mathrm{PAH}$ feature.

on the PAH feature strength in the range of inclinations where the line-of-sight passes from through the envelope to through the outflow cavity. Strong PAH features are predicted to occur when observing down the outflow cavity. This situation can be recognized by a strong UV and optical part of the SED, as well as a lack of absorption features. For typical values for the luminosity, UV excess, inclination and envelope mass, derived from observational studies of embedded protostars, and assuming an ISM abundance, the PAH features should be detectable. The low detection rate of PAHs toward these sources thus suggests that the abundance of PAHs is at least 20-50x lower than in the ISM. Decreasing luminosity, UV excess or envelope mass compared with the template model changes this conclusion to a typical factor of 10-20, compared with the ISM abundance.

One caveat remains with respect to the effect of scattering. The radiative transfer code used here assumes all scattering to be isotropic. This does not allow us to treat the effect of preferentially forward scattering of UV radiation through the outflow cavity. In the current implementation, our models could overestimate the amount of radiation received by the disk as well as overestimate the strength of the PAH features. Thus, our inferred abundance limits are on the low side. As a second caveat, an external interstellar radiation field is not included in these models. Interstellar UV radiation from outside the embedded object may become a significant source of excitation for PAHs in the envelope at large radii and would increase the predicted PAH feature strength and thus constrain the PAH abundance upper limits to smaller values, going in opposite directions.

\subsection{PAH evolution from clouds to disks}

In both class I and II low-mass objects that we have studied, we find very low detection rates for PAHs. Comparison with model predictions suggest that for typical conditions in class I protostars, the absence of exciting radiation and attenuation by foreground material can be excluded as reason for the absence of the PAH features. In addition, the predicted feature/continuum ratio is well within the achieved sensitivity of the observations. This thus implies an absence of carrier as the reason the for absence of features, and the PAH abundance in the embedded protostar phase is estimated here to be at least a factor of 10-20 times lower than for typical (number of carbon atoms $N_{C}=100$ ) PAHs in the ISM. This lower abundance is in the same range as those inferred toward T Tauri stars in Geers et al. (2006). Thus, even though the detection rate in the Class I objects is even lower than that found toward disks, the inferred abundances are comparable. Note that the few Class II disks in which we detect PAHs are mostly G-stars. The disk sample in Geers et al. (2006) is more biased toward higher mass stars.

What would cause this lower abundance in the embedded phase, and is this mechanism the same in the class II phase? In a cold dense environment, two possibilities for lowering the abundance of a species are recognized: coagulation or dust growth and freeze-out onto larger grains.

The freeze-out of PAHs onto grains could explain the lower PAH abundance in these objects. PAH features in absorption have been observed in the ISM, and laboratory spectra of PAHs condensed into $\mathrm{H}_{2} \mathrm{O}$ rich ices suggest that PAH absorption features should also be observable in astrophysical ices, such as in dense clouds (Bernstein et al. 2007, and references therein). During this phase, if exposed to UV radiation, PAHs in water ice can become significantly ionized, thus promoting ionmediated reactions in these ices with implications for astrochemistry (Ehrenfreund et al. 2006; Gudipati \& Allamandola 2006). In the class II phase, the direct irradiation of the disk by the central star can increase the temperature in the surface layers of the disk, evaporating the ice and depositing the enclosed PAHs back into the gas, thereby increasing the abundance of the PAHs again. Recent observations of edge-on disks show evidence for the continued presence of a reservoir of ice in the interior of disks (Pontoppidan et al. 2005; Terada et al. 2007) which can be brought to the surface by vertical mixing. If the PAHs in the ices react to form larger carbonaceous materials, then only a fraction of PAHs might come back off the grain when the ice layer evaporates. This could be a possible explanation for the low PAH abundance inferred for the majority of T Tauri disk surface layers. In a study of the ice features of the same c2d sample of embedded sources, Boogert et al. (2008) show spectra over the 5-10 $\mu \mathrm{m}$ range where absorptions due to PAHs in ices occur. No obvious features are detected, but the laboratory spectroscopy is not well known and some PAH bands could be hidden in the $6 \mu \mathrm{m}$ complex, especially on the long wavelength side of the C2 component in Boogert et al. (2008). Assuming a typical (but uncertain) oscillator strength for PAHs, up to $10 \%$ of the carbon could be locked up in PAHs in the ices (Boogert, priv. comm.). These limits are consistent with the amount of freeze-out found in this work.

PAHs growing and/or agglomerating to typically 100x larger particles could be an alternative explanation for the lower PAH abundance. This process could take place in the high density regions of the disk and envelope, in both the class I and II phase, and does not exclude freeze-out as an option. Observationally, the presence of broad emission plateaus between 6-9, 11-13 and $15-20 \mu \mathrm{m}$ has been attributed to large 200-2000 carbon atom PAHs and PAH clusters (Allamandola et al. 1989; Van Kerckhoven et al. 2000), which will lose their characteristic PAH emission features, thus additionally lowering the 
feature/continuum of these features. The detection of individual features toward T Tauri disks shows that this process is not necessarily dominant, and that either small PAHs are still released from the ice in this phase and/or that small PAHs are still being created from, e.g., the destruction of larger grains or agglomerates through collisions.

\section{Conclusions}

No PAHs are detected toward any of the 53 confirmed embedded sources in either our Spitzer or ISAAC surveys. PAHs are detected at $3.3 \mu \mathrm{m}$ towards 1 of the 17 confirmed disk sources, IRS 48 , and at $11.2 \mu \mathrm{m}$ for 1 of the 10 sources with an uncertain or borderline embedded classification, Oph GY 23. For all 12 sources with ISAAC and Spitzer spectra, no PAH features are detected in either. In total, PAH features are detected toward at most 1 out of 63 (candidate) embedded protostars (1.6\%), even lower than observed for class II T Tauri stars with disks $(11-14 \%)$.

The low PAH detection rate is compared with radiative transfer model calculations. It is shown that the effects of envelope mass on the absorption of PAH features is not enough to obscure PAH features. The $7.7 \mu \mathrm{m}$ feature is predicted by models as the best tracer of PAH emission, while the $3.3 \mu \mathrm{m}$ feature is relatively weak.

Assuming typical class I stellar and envelope parameters, the absence of PAH emission is most likely explained by the absence of emitting carriers through a much lower PAH abundance in the gas, e.g., due to freeze-out of PAHs on icy layers on dust grains or agglomeration. Our inferred low abundances are similar to those found for disks around T Tauri stars. Thus PAHs are expected to be removed from the gas at earlier stages of star- and planet formation and enter the disks frozen out on grains. Further searches for PAH absorption features are needed to constrain the presence of PAHs in icy grains.

Acknowledgements. The authors thank R. Visser for his models and comments. Support for this work, part of the Spitzer Legacy Science Program, was provided by NASA through contracts 1224608, 1230779 and 1256316 issued by the Jet Propulsion Laboratory, California Institute of Technology, under NASA contract 1407. A.C. was supported by a fellowship from the European Research Training Network "The Origin of Planetary Systems" (PLANETS, contract number HPRN-CT-2002-00308) at Leiden Observatory and by a Marie Curie Intra-European Fellowship from the European Community (contract number FP6-024227) at Observatorio Astronómico Nacional. Astrochemistry in Leiden is supported by a NWO Spinoza grant and a NOVA grant.

\section{References}

Acke, B., \& van den Ancker, M. E. 2004, A\&A, 426, 151

Allamandola, L. J., Tielens, G. G. M., \& Barker, J. R. 1989, ApJS, 71, 733

Arce, H. G., \& Sargent, A. I. 2006, ApJ, 646, 1070

Basri, G., \& Bertout, C. 1989, ApJ, 341, 340

Bernstein, M. P., Sandford, S. A., Allamandola, L. J., et al. 1999, Science, 283, 1135

Bernstein, M. P., Sandford, S. A., Mattioda, A. L., \& Allamandola, L. J. 2007, ApJ, 664, 1264
Boogert, A. C. A., Pontoppidan, K. M., Knez, C., et al. 2008, ApJ, 678, 985 Calvet, N., \& Gullbring, E. 1998, ApJ, 509, 802

Chiang, E. I., \& Goldreich, P. 1997, ApJ, 490, 368

Crapsi, A., van Dishoeck, E. F., Hogerheijde, M. R., Pontoppidan, K. M., \& Dullemond, C. P. 2008, A\&A, 486, 245

Draine, B. T., \& Li, A. 2001, ApJ, 551, 807

Dullemond, C. P., \& Dominik, C. 2004, A\&A, 417, 159

Dullemond, C. P., \& Turolla, R. 2000, A\&A, 360, 1187

Ehrenfreund, P., Rasmussen, S., Cleaves, J., \& Chen, L. 2006, Astrobiology, 6, 490

Enoch, M. L., Evans, II, N. J., Sargent, A. I., et al. 2008, ApJ, accepted, [arXiv/0805.1075]

Evans, II, N. J., Allen, L. E., Blake, G. A., et al. 2003, PASP, 115, 965

Evans, II, N. J., Dunham, M. M., Jørgensen, J. K., et al. 2008, ApJ in press [arXiv: 0811. 1059]

Geers, V. C. 2007, Ph.D. in Astronomy, Leiden Observatory - Leiden University, PO Box 9513, 2300 (RA Leiden, The Netherlands)

Geers, V. C., Augereau, J.-C., Pontoppidan, K. M., et al. 2006, A\&A, 459, 545

Geers, V. C., Pontoppidan, K. M., van Dishoeck, E. F., et al. 2007a, A\&A, 469, L35

Geers, V. C., van Dishoeck, E. F., Visser, R., et al. 2007b, A\&A, 476, 279

Gudipati, M. S., \& Allamandola, L. J. 2006, ApJ, 638, 286

Habart, E., Natta, A., \& Krügel, E. 2004, A\&A, 427, 179

Habart, E., Natta, A., Testi, L., \& Carbillet, M. 2006, A\&A, 449, 1067

Hartmann, L. W., \& Kenyon, S. J. 1990, ApJ, 349, 190

Helling, C., Jorgensen, U. G., Plez, B., \& Johnson, H. R. 1996, A\&A, 315, 194

Hogerheijde, M. R., van Dishoeck, E. F., Blake, G. A., \& van Langevelde, H. J. 1998, ApJ, 502, 315

Jonkheid, B., Kamp, I., Augereau, J.-C., \& van Dishoeck, E. F. 2006, A\&A, 453, 163

Jørgensen, J. K., Johnstone, D., Kirk, H., et al. 2008, ApJ, 683, 822

Kraus, S., Hofmann, K.-H., Benisty, M., et al. 2008, A\&A, 489, 1157

Lahuis, F., Kessler-Silacci, J. E., Evans, II, N. J., et al. 2006, c2d Spectroscopy

Explanatory Supplement (Pasadena: Spitzer Science Center)

Lahuis, F., van Dishoeck, E. F., Blake, G. A., et al. 2007, ApJ, 665, 492

Luhman, K. L. 2007, ApJS, 173, 104

Manske, V., \& Henning, T. 1999, A\&A, 349, 907

Mattioda, A. L., Hudgins, D. M., \& Allamandola, L. J. 2005, ApJ, 629, 1188

Oliveira, I., Merín, B., Pontoppidan, K. M., et al. 2008, ApJ, in press [arXiv: 0810.0829]

Peeters, E., Hony, S., Van Kerckhoven, C., et al. 2002, A\&A, 390, 1089

Pontoppidan, K. M., Fraser, H. J., Dartois, E., et al. 2003, A\&A, 408, 981

Pontoppidan, K. M., Dullemond, C. P., van Dishoeck, E. F., et al. 2005, ApJ, 622,463

Pontoppidan, K. M., Boogert, A. C. A., Fraser, H. J., et al. 2008, ApJ, 678, 1005 Ressler, M. E., \& Barsony, M. 2003, ApJ, 584, 832

Siebenmorgen, R., Kruegel, E., \& Mathis, J. S. 1992, A\&A, 266, 501

Terada, H., Tokunaga, A. T., Kobayashi, N., et al. 2007, ApJ, 667, 303

Thi, W.-F., van Dishoeck, E. F., Dartois, E., et al. 2006, A\&A, 449, 251

Ulrich, R. K. 1976, ApJ, 210, 377

van Dishoeck, E. F., \& Black, J. H. 1982, ApJ, 258, 533

van Dishoeck, E. F., \& van der Tak, F. F. S. 2000, From Molecular Clouds to

Planetary Systems, ed. Y. C. Minh, \& E. F. van Dishoeck (San Francisco: ASP), IAU Symp., 197, 97

van Dishoeck, E. F., Dartois, E., Pontoppidan, K. M., et al. 2003, The Messenger, 113,49

van Kempen, T. A. 2008, Ph.D. in Astronomy, Leiden Observatory - Leiden University, PO Box 9513, 2300 RA Leiden, The Netherlands

van Kempen, T. A., van Dishoeck, E. F., Salter, D. M., et al. 2008, A\&A, submitted

Van Kerckhoven, C., Hony, S., Peeters, E., et al. 2000, A\&A, 357, 1013

Verstraete, L., Puget, J. L., Falgarone, E., et al. 1996, A\&A, 315, L337

Visser, R., Geers, V. C., Dullemond, C. P., et al. 2007, A\&A, 466, 229

Weingartner, J. C., \& Draine, B. T. 2001, ApJ, 548, 296

Whitney, B. A., Wood, K., Bjorkman, J. E., \& Wolff, M. J. 2003, ApJ, 591, 1049 
Appendix A: Table: summary of observations

Table A.1. Summary of observations. Line flux in $\mathrm{W} \mathrm{m}^{-2}$.

\begin{tabular}{|c|c|c|c|c|c|}
\hline Target & $\begin{array}{l}\text { RA } \\
{[\mathrm{J} 2000]} \\
\end{array}$ & $\begin{array}{l}\text { Dec } \\
{[\mathrm{J} 2000]}\end{array}$ & class & $\begin{array}{l}\text { Line flux } \\
3.3 \mu \mathrm{m} \\
\end{array}$ & Comments \\
\hline \multicolumn{6}{|l|}{ Perseus } \\
\hline LDN1448 IRS1 & $3^{\mathrm{h}} 25^{\mathrm{m}} 09 \mathrm{~s} .4$ & $+30^{\circ} 46^{\prime} 21^{\prime \prime} .7$ & embedded & - & Spitzer \\
\hline LDN1448 NA & $3^{\mathrm{h}} 25^{\mathrm{m}} 36.5$ & $+30^{\circ} 45^{\prime} 21^{\prime \prime} .2$ & embedded & - & Spitzer \\
\hline IRAS $03245+3002$ & $3^{\mathrm{h}} 27^{\mathrm{m}} 39^{\mathrm{s}} .0$ & $+30^{\circ} 12^{\prime} 59^{\prime} .4$ & embedded & - & Spitzer \\
\hline L1455 SMM1 & $3^{\mathrm{h}} 27^{\mathrm{m}} 43^{\mathrm{s}} .2$ & $+30^{\circ} 12^{\prime} 28^{\prime \prime} .8$ & embedded & - & Spitzer \\
\hline L1455 IRS3 & $3^{\mathrm{h}} 28^{\mathrm{m}} 00^{\mathrm{s}} .4$ & $+30^{\circ} 08^{\prime} 01^{\prime \prime} 3$ & embedded & - & Spitzer \\
\hline IRAS $03254+3050$ & $3^{\mathrm{h}} 28^{\mathrm{m}} 34.5$ & $+31^{\circ} 00^{\prime} 51^{\prime \prime} .1$ & embedded & - & Spitzer \\
\hline IRAS $03271+3013$ & $3^{\mathrm{h}} 30^{\mathrm{m}} 15^{\mathrm{s}} .2$ & $+30^{\circ} 23^{\prime} 48^{\prime \prime} .8$ & embedded & - & Spitzer \\
\hline IRAS 03301+3111 & $3^{\mathrm{h}} 33^{\mathrm{m}} 12^{\mathrm{s}} .8$ & $+31^{\circ} 21^{\prime} 24^{\prime \prime} .1$ & embedded & - & Spitzer \\
\hline B1-a & $3^{\mathrm{h}} 33^{\mathrm{m}} 16^{\mathrm{s}} .7$ & $+31^{\circ} 07^{\prime} 55^{\prime \prime} .2$ & embedded & - & Spitzer \\
\hline $\mathrm{B} 1-\mathrm{c}$ & $3^{\mathrm{h}} 33^{\mathrm{m}} 17^{\mathrm{s}} .9$ & $+31^{\circ} 09^{\prime} 31^{\prime \prime} 0$ & embedded & - & Spitzer \\
\hline SSTc2d J033327.3+310710 & $3^{\mathrm{h}} 33^{\mathrm{m}} 27^{\circledR} .3$ & $+31^{\circ} 07^{\prime} 10^{\prime} .2$ & embedded & - & Spitzer \\
\hline HH 211-mm & $3^{\text {h }} 43^{\mathrm{m}} 56^{\mathrm{s}} .8$ & $+32^{\circ} 00^{\prime} 50^{\prime} .4$ & embedded & - & Spitzer \\
\hline IRAS $03439+3233$ & $3^{\mathrm{h}} 47^{\mathrm{m}} 05^{\mathrm{s}} .4$ & $+32^{\circ} 43^{\prime} 08^{\prime \prime} .4$ & embedded/borderline & - & Spitzer \\
\hline IRAS $03445+3242$ & $3^{\mathrm{h}} 47^{\mathrm{m}} 41^{\mathrm{s}} .6$ & $+32^{\circ} 51^{\prime} 43^{\prime \prime} .9$ & embedded & - & Spitzer \\
\hline \multicolumn{6}{|l|}{ Taurus } \\
\hline LDN 1489 IRS & $04^{\mathrm{h}} 04^{\mathrm{m}} 42^{\mathrm{s}} .9$ & $+26^{\circ} 18^{\prime} 56^{\prime \prime} 3$ & embedded & $\leq 8.8 \mathrm{E}-16$ & ISAAC \\
\hline \multicolumn{6}{|l|}{ Orion } \\
\hline Reipurth 50 & $05^{\mathrm{h}} 40^{\mathrm{m}} 27^{\mathrm{s}} .7$ & $-07^{\circ} 27^{\prime} 32^{\prime \prime} .1$ & embedded & $\leq 1.0 \mathrm{E}-15$ & ISAAC \\
\hline TPSC 78 & $05^{\mathrm{h}} 35^{\mathrm{m}} 14^{\mathrm{s}} .1$ & $-05^{\circ} 23^{\prime} 38^{\prime \prime} .4$ & uncertain & $\leq 2.3 \mathrm{E}-16$ & ISAAC \\
\hline TPSC 1 & $05^{\mathrm{h}} 35^{\mathrm{m}} 14^{\mathrm{s}} .5$ & $-05^{\circ} 23^{\prime} 54^{\prime \prime} .7$ & uncertain & $\leq 1.0 \mathrm{E}-16$ & ISAAC \\
\hline \multicolumn{6}{|l|}{ Vela } \\
\hline HH 46 / IRAS 08242-5050 & $08^{\mathrm{h}} 25^{\mathrm{m}} 43^{\mathrm{s}} .8$ & $-51^{\circ} 00^{\prime} 35^{\prime \prime} .6$ & embedded & $\leq 1.2 \mathrm{E}-16$ & ISAAC, Spitzer \\
\hline IRAS 08261-5100 & $08^{\mathrm{h}} 27^{\mathrm{m}} 38^{\mathrm{s}} .9$ & $-51^{\circ} 10^{\prime} 37^{\prime \prime} .2$ & embedded & - & Spitzer \\
\hline LLN 20 & $08^{\mathrm{h}} 47^{\mathrm{m}} 39^{\mathrm{s}} .4$ & $-43^{\circ} 06^{\prime} 08^{\prime \prime}-$ & embedded & $\leq 1.1 \mathrm{E}-16$ & ISAAC \\
\hline LLN 33 & $08^{\mathrm{h}} 57^{\mathrm{m}} 36^{\mathrm{s}} .8$ & $-43^{\circ} 14^{\prime} 35^{\prime \prime}-$ & embedded & $\leq 1.3 \mathrm{E}-16$ & ISAAC \\
\hline LLN 47 & $09^{\mathrm{h}} 09^{\mathrm{m}} 25^{\mathrm{s}} 6$ & $-45^{\circ} 22^{\prime} 51^{\prime \prime}-$ & embedded & $\leq 5.3 \mathrm{E}-16$ & ISAAC \\
\hline \multicolumn{6}{|l|}{ Chamaeleon } \\
\hline Ced 110 IRS4 & $11^{\mathrm{h}} 06^{\mathrm{m}} 46^{\mathrm{s}} .6$ & $-77^{\circ} 22^{\prime} 32^{\prime \prime} .5$ & embedded & & Spitzer \\
\hline IRAS 11068-7717 & $11^{\mathrm{h}} 08^{\mathrm{m}} 15^{\mathrm{s}} .1$ & $-77^{\circ} 33^{\prime} 53^{\prime \prime} .2$ & uncertain & $\leq 5.5 \mathrm{E}-15$ & ISAAC \\
\hline Cha IRN & $11^{\mathrm{h}} 08^{\mathrm{m}} 38^{\mathrm{s}} .2$ & $-77^{\circ} 43^{\prime} 51^{\prime \prime} .7$ & embedded & $\leq 4.2 \mathrm{E}-16$ & ISAAC \\
\hline Cha INa 2 & $11^{\mathrm{h}} 09^{\mathrm{m}} 36^{\mathrm{s}} .6$ & $-76^{\circ} 33^{\prime} 39^{\prime}$. & disk & $\leq 5.1 \mathrm{E}-16$ & ISAAC \\
\hline IRAS 12553-7651 & $12^{\mathrm{h}} 59^{\mathrm{m}} 06^{\mathrm{s}} .6$ & $-77^{\circ} 07^{\prime} 40^{\prime} .1$ & embedded & & Spitzer \\
\hline \multicolumn{6}{|l|}{ Ophiuchus } \\
\hline VSSG 1 & $16^{\mathrm{h}} 26^{\mathrm{m}} 18^{\mathrm{s}} .8$ & $-24^{\circ} 28^{\prime} 49^{\prime} .5$ & disk & $\leq 9.5 \mathrm{E}-16$ & ISAAC, Spitzer \\
\hline GSS30 IRS 1 & $16^{\mathrm{h}} 26^{\mathrm{m}} 21^{\mathrm{s}} .4$ & $-24^{\circ} 23^{\prime} 04^{\prime \prime} .2$ & embedded & $\leq 8.6 \mathrm{E}-16$ & ISAAC, Spitzer \\
\hline Oph GY 23 & $16^{\mathrm{h}} 26^{\mathrm{m}} 24^{\mathrm{s}} .1$ & $-24^{\circ} 24^{\prime} 48^{\prime \prime} .2$ & embedded/borderline & - & Spitzer \\
\hline VLA 1623-2418 & $16^{\mathrm{h}} 26^{\mathrm{m}} 26^{\mathrm{s}} .4$ & $-24^{\circ} 24^{\prime} 30^{\prime} .2$ & embedded & - & Spitzer \\
\hline IRS 14 & $16^{\mathrm{h}} 26^{\mathrm{m}} 31^{\mathrm{s}} .0$ & $-24^{\circ} 31^{\prime} 05^{\prime \prime} .2$ & disk & - & Spitzer \\
\hline WL 12 & $16^{\mathrm{h}} 26^{\mathrm{m}} 44^{\mathrm{s}} .2$ & $-24^{\circ} 34^{\prime} 48^{\prime \prime} .4$ & embedded & $\leq 2.6 \mathrm{E}-16$ & ISAAC, Spitzer \\
\hline OphE-MM3 & $16^{\mathrm{h}} 27^{\mathrm{m}} 05^{\mathrm{s}} .9$ & $-24^{\circ} 37^{\prime} 08^{\prime \prime} 0$ & disk & - & Spitzer \\
\hline GY 224 & $16^{\mathrm{h}} 27^{\mathrm{m}} 11^{\mathrm{s}} .2$ & $-24^{\circ} 40^{\prime} 46^{\prime \prime} 6$ & disk & - & Spitzer \\
\hline WL 19 & $16^{\mathrm{h}} 27^{\mathrm{m}} 11^{\mathrm{s}} .7$ & $-24^{\circ} 38^{\prime} 32^{\prime \prime} 3$ & uncertain & - & Spitzer \\
\hline WL 20S & $16^{\mathrm{h}} 27^{\mathrm{m}} 15^{\mathrm{s}} .6$ & $-24^{\circ} 38^{\prime} 45^{\prime \prime} 6$ & disk & $\leq 2.0 \mathrm{E}-16$ & ISAAC, Spitzer \\
\hline WL 20E & $16^{\mathrm{h}} 27^{\mathrm{m}} 15^{\mathrm{s}} .7$ & $-24^{\circ} 38^{\prime} 59^{\prime} .8$ & disk & $\leq 2.1 \mathrm{E}-16$ & ISAAC \\
\hline IRS 37 & $16^{\mathrm{h}} 27^{\mathrm{m}} 17^{\mathrm{s}} .6$ & $-24^{\circ} 28^{\prime} 56^{\prime \prime} 6$ & embedded & - & Spitzer \\
\hline IRS 42 & $16^{\mathrm{h}} 27^{\mathrm{m}} 21^{\mathrm{s}} .5$ & $-24^{\circ} 41^{\prime} 43^{\prime \prime} .1$ & disk & $\leq 2.0 \mathrm{E}-15$ & ISAAC \\
\hline WL 6 & $16^{\mathrm{h}} 27^{\mathrm{m}} 21^{\mathrm{s}} .8$ & $-24^{\circ} 29^{\prime} 53^{\prime \prime} .2$ & embedded & - & Spitzer \\
\hline CRBR 2422.8-3423 & $16^{\mathrm{h}} 27^{\mathrm{m}} 24^{\mathrm{s}} .6$ & $-24^{\circ} 41^{\prime} 03^{\prime \prime} .1$ & disk & $\leq 8.7 \mathrm{E}-16$ & ISAAC, Spitzer \\
\hline IRS 43 & $16^{\mathrm{h}} 27^{\mathrm{m}} 26^{\mathrm{s}} .9$ & $-24^{\circ} 40^{\prime} 50^{\prime} 8$ & embedded & $\leq 4.4 \mathrm{E}-15$ & ISAAC \\
\hline IRS 44 & $16^{\mathrm{h}} 27^{\mathrm{m}} 28^{\mathrm{s}} 0$ & $-24^{\circ} 39^{\prime} 33^{\prime \prime} .5$ & embedded & $\leq 1.3 \mathrm{E}-15$ & ISAAC \\
\hline Elias 32 & $16^{\mathrm{h}} 27^{\mathrm{m}} 28^{\mathrm{s}} .4$ & $-24^{\circ} 27^{\prime} 21^{\prime \prime} .2$ & embedded & $\leq 8.4 \mathrm{E}-16$ & ISAAC, Spitzer \\
\hline IRS 46 & $16^{\mathrm{h}} 27^{\mathrm{m}} 29^{\mathrm{s}} .4$ & $-24^{\circ} 39^{\prime} 16^{\prime \prime} \cdot 2$ & disk & $\leq 1.4 \mathrm{E}-15$ & ISAAC, Spitzer \\
\hline VSSG 17 & $16^{\mathrm{h}} 27^{\mathrm{m}} 30^{\mathrm{s}} .2$ & $-24^{\circ} 27^{\prime} 44^{\prime \prime} 3$ & embedded & $\leq 3.9 \mathrm{E}-16$ & ISAAC, Spitzer \\
\hline IRS 48 & $16^{\mathrm{h}} 27^{\mathrm{m}} 37^{\mathrm{s}} .2$ & $-24^{\circ} 30^{\prime} 35^{\prime \prime} 0$ & disk & $3.0 \mathrm{E}-15$ & ISAAC \\
\hline IRS 51 & $16^{\mathrm{h}} 27^{\mathrm{m}} 39^{\mathrm{s}} .8$ & $-24^{\circ} 43^{\prime} 15^{\prime \prime} .1$ & disk & $\leq 9.2 \mathrm{E}-16$ & ISAAC \\
\hline IRS 54 & $16^{\mathrm{h}} 27^{\mathrm{m}} 51^{\mathrm{s}} .8$ & $-24^{\circ} 31^{\prime} 45^{\prime \prime} .5$ & disk & $\leq 3.8 \mathrm{E}-16$ & ISAAC \\
\hline IRS 63 & $16^{\mathrm{h}} 31^{\mathrm{m}} 35^{\mathrm{s}} .7$ & $-24^{\circ} 01^{\prime} 29^{\prime} .6$ & embedded & $\leq 3.2 \mathrm{E}-15$ & ISAAC, Spitzer \\
\hline L1689-IRS5 & $16^{\mathrm{h}} 31^{\mathrm{m}} 52^{\mathrm{s}} .1$ & $-24^{\circ} 56^{\prime} 15^{\prime \prime} 4$ & uncertain & - & Spitzer \\
\hline IRAS 16293-2422B & $16^{\mathrm{h}} 32^{\mathrm{m}} 22^{\mathrm{s}} .6$ & $-24^{\circ} 28^{\prime} 32^{\prime \prime} .2$ & embedded & - & Spitzer \\
\hline IRAS 16293-2422 & $16^{\mathrm{h}} 32^{\mathrm{m}} 22^{\mathrm{s}} .9$ & $-24^{\circ} 28^{\prime} 36^{\prime \prime}{ }^{\prime} 1$ & embedded & - & Spitzer \\
\hline RNO 91 & $16^{\mathrm{h}} 34^{\mathrm{m}} 29^{\mathrm{s}} 3$ & $-15^{\circ} 47^{\prime} 01^{\prime \prime} 3$ & embedded & - & Spitzer \\
\hline
\end{tabular}


Table A.1. continued.

\begin{tabular}{|c|c|c|c|c|c|}
\hline Target & $\begin{array}{l}\text { RA } \\
{[\mathrm{J} 2000]}\end{array}$ & $\begin{array}{l}\text { Dec } \\
{[\mathrm{J} 2000]}\end{array}$ & class & $\begin{array}{l}\text { Line flux } \\
3.3 \mu \mathrm{m}\end{array}$ & Comments \\
\hline \multicolumn{6}{|l|}{ Serpens } \\
\hline SSTc2d J182901.8+02954 & $18^{\mathrm{h}} 29^{\mathrm{m}} 01^{\mathrm{s}} .8$ & $+00^{\circ} 29^{\prime} 54^{\prime \prime} .2$ & uncertain & - & Spitzer \\
\hline SSTc2d J182916.2+01822 & $18^{\mathrm{h}} 29^{\mathrm{m}} 16^{\mathrm{s}} .2$ & $+00^{\circ} 18^{\prime} 22^{\prime \prime} .7$ & embedded & - & Spitzer \\
\hline Serp-S68N & $18^{\mathrm{h}} 29^{\mathrm{m}} 48^{\mathrm{s}} .1$ & $+01^{\circ} 16^{\prime} 42^{\prime \prime} .6$ & embedded & - & Spitzer \\
\hline EC 69 & $18^{\mathrm{h}} 29^{\mathrm{m}} 54.4$ & $+01^{\circ} 15^{\prime} 01^{\prime \prime} .8$ & uncertain & - & Spitzer \\
\hline SVS 4-2 & $18^{\mathrm{h}} 29^{\mathrm{m}} 56^{\mathrm{s}} .6$ & $+01^{\circ} 12^{\prime} 59^{\prime} 4$ & disk & $\leq 6.4 \mathrm{E}-17$ & ISAAC \\
\hline Serp-SMM4 & $18^{\mathrm{h}} 29^{\mathrm{m}} 56^{\mathrm{s}} .6$ & $+01^{\circ} 13^{\prime} 15^{\prime \prime} .2$ & embedded & - & Spitzer \\
\hline EC 82 & $18^{\mathrm{h}} 29^{\mathrm{m}} 56^{\mathrm{s}} .9$ & $+01^{\circ} 14^{\prime} 46^{\prime \prime} .4$ & disk & $\leq 4.9 \mathrm{E}-16$ & ISAAC \\
\hline EC 90A & $18^{\mathrm{h}} 29^{\mathrm{m}} 57^{\mathrm{s}} .3$ & $+01^{\circ} 14^{\prime} 03^{\prime \prime} .7$ & embedded & $\leq 2.1 \mathrm{E}-15$ & ISAAC \\
\hline EC 90B & $18^{\mathrm{h}} 29^{\mathrm{m}} 57^{\mathrm{s}} .3$ & $+01^{\circ} 14^{\prime} 03^{\prime \prime} .7$ & embedded & $\leq 7.4 \mathrm{E}-16$ & ISAAC \\
\hline EC 88 & $18^{\mathrm{h}} 29^{\mathrm{m}} 57^{\mathrm{s}} .6$ & $+01^{\circ} 13^{\prime} 00^{\prime} .5$ & embedded & - & Spitzer \\
\hline SVS 4-3 & $18^{\mathrm{h}} 29^{\mathrm{m}} 56^{\mathrm{s}} .7$ & $+01^{\circ} 12^{\prime} 39^{\prime} 0$ & disk & $\leq 1.4 \mathrm{E}-16$ & ISAAC \\
\hline SVS 4-5 & $18^{\mathrm{h}} 29^{\mathrm{m}} 57^{\mathrm{s}} .6$ & $+01^{\circ} 13^{\prime} 00^{\prime} .^{\prime}$ & disk & $\leq 1.6 \mathrm{E}-16$ & ISAAC \\
\hline SVS 4-9 & $18^{\mathrm{h}} 29^{\mathrm{m}} 58^{\mathrm{s}} .1$ & $+01^{\circ} 12^{\prime} 39^{\prime} 4$ & disk & $\leq 3.2 \mathrm{E}-16$ & ISAAC \\
\hline Serp-SMM3 & $18^{\mathrm{h}} 29^{\mathrm{m}} 59^{\mathrm{s}} .2$ & $+01^{\circ} 14^{\prime} 00^{\prime} \cdot 2$ & embedded & - & Spitzer \\
\hline \multicolumn{6}{|l|}{ Coronis Australis } \\
\hline R CrA IRS 5A & $19^{\mathrm{h}} 01^{\mathrm{m}} 48.1$ & $-36^{\circ} 57^{\prime} 21^{\prime \prime} .9$ & embedded & $\leq 4.2 \mathrm{E}-16$ & ISAAC \\
\hline R CrA IRS 5B & $19^{\mathrm{h}} 01^{\mathrm{m}} 48^{\mathrm{s}} .1$ & $-36^{\circ} 57^{\prime} 21^{\prime \prime} .9$ & embedded & $\leq 1.7 \mathrm{E}-16$ & ISAAC \\
\hline $\mathrm{R}$ CrA IRS $5 \mathrm{~A}+\mathrm{B}$ & $19^{\mathrm{h}} 01^{\mathrm{m}} 48^{\mathrm{s}} .0$ & $-36^{\circ} 57^{\prime} 21^{\prime \prime} 6$ & embedded & - & Spitzer \\
\hline HH 100 IRS & $19^{\mathrm{h}} 01^{\mathrm{m}} 50.7$ & $-36^{\circ} 58^{\prime} 09^{\prime} 6$ & embedded & $\leq 8.8 \mathrm{E}-15$ & ISAAC \\
\hline R CrA IRS 7A & $19^{\mathrm{h}} 01^{\mathrm{m}} 55^{\mathrm{s}} .3$ & $-36^{\circ} 57^{\prime} 22^{\prime \prime} .0$ & embedded & $\leq 1.5 \mathrm{E}-16$ & ISAAC, Spitzer \\
\hline R CrA IRS 7B & $19^{\mathrm{h}} 01^{\mathrm{m}} 56^{\mathrm{s}} .4$ & $-36^{\circ} 57^{\prime} 28^{\prime \prime} .1$ & embedded/borderline & $\leq 7.1 \mathrm{E}-17$ & ISAAC, Spitzer \\
\hline R CrA IRAS32 & $19^{\mathrm{h}} 02^{\mathrm{m}} 58^{\mathrm{s}} .7$ & $-37^{\circ} 07^{\prime} 34^{\prime \prime} .7$ & embedded & - & Spitzer \\
\hline \multicolumn{6}{|l|}{ Additional sources } \\
\hline IRAS 13546-3941 & $13^{\mathrm{h}} 57^{\mathrm{m}} 38^{\mathrm{s}} .9$ & $-39^{\circ} 56^{\prime} 00^{\prime} .^{2}$ & disk & - & Spitzer \\
\hline IRAS 15398-3359 & $15^{\mathrm{h}} 43^{\mathrm{m}} 02^{\mathrm{s}} .3$ & $-34^{\circ} 09^{\prime} 06^{\prime \prime} 8$ & embedded & - & Spitzer \\
\hline IRAS $23238+7401$ & $23^{\mathrm{h}} 25^{\mathrm{m}} 46^{\mathrm{s}} .7$ & $+74^{\circ} 17^{\prime} 37^{\prime \prime} .3$ & embedded & - & Spitzer \\
\hline
\end{tabular}

